\title{
Osteoporose - Diagnostik und Therapie
}

\author{
Tobias Vogel, Philip Kampmann, Rainer Bartl, Klaus Jürgen Pfeifer, Wolf Mutschler
}

\section{Zusammenfassung}

In Deutschland ist die Osteoporose immer noch eine unterschätzte, unterdiagnostizierte und untertherapierte Krankheit. Sie wird erst nach dem Auftreten von Frakturen klinisch relevant, denn Knochenschwund alleine verursacht in der Regel weder Schmerzen noch Bewegungseinschränkung. Durch eine leitliniengerechte Diagnostik und Therapie lassen sich die häufigen Folgefrakturen effektiv reduzieren und die Lebensqualität der betroffenen Patienten entscheidend verbessern. Die evidenzbasierte Diagnostik und Therapie der Osteoporose ist anhand internationaler wie nationaler Leitlinien sicher und einfach durchführbar. Mittels konventioneller Röntgenaufnahmen und Dual-X-ray-Absoptiometrie (DXA) ist eine Diagnosestellung schonend, schnell und kostengünstig durchzuführen. Bisphosphonate, selektive Östrogen-Rezeptormodulatoren und andere Medikamente haben ihre Wirksamkeit bezüglich einer Senkung des Folgefrakturrisikos in großen randomisiert-kontrollierten Studien bewiesen. Was allerdings heute noch fehlt ist eine konsequente Umsetzung dieser Erkenntnisse durch die an der Frakturversorgung beteiligten Ärzte.

\section{Einleitung}

„Gelenkerkrankungen, Rückenbeschwerden, Osteoporose und unfallbedingte Extremitätenverletzungen haben einen enormen Einfluss auf den Einzelnen und die Gesellschaft sowie auf das Gesundheitswesen und die Volkswirtschaft. Es existieren effektive Möglichkeiten zur Prävention und Behandlung dieser entstellenden Leiden, aber wir müssen jetzt handeln“. Mit diesen Worten erklärte UN-Generalsekretär Kofi Annan zusammen mit der Weltgesundheitsorganisation (WHO) die erste Dekade des neuen Jahrtausend zur „Bone and Joint Decade“ [4]. Seither wird der Osteoporose durch die Bevölkerung, die betroffenen Patienten und die behandelnden Ärzte in zunehmendem Maße Beachtung geschenkt.

OP-JOURNAL 2005; 21: 154-159

(c) Georg Thieme Verlag KG Stuttgart · New York
Die WHO definiert die Osteoporose als „....eine systemische Erkrankung des Skeletts, die durch eine erniedrigte Knochenmasse und eine Verschlechterung der Mikroarchitektur des Knochens gekennzeichnet ist. Daraus folgt eine zunehmende Brüchigkeit und ein gehäuftes Auftreten von Frakturen“" [6].

Die Einteilung der Osteoporose erfolgt nach unterschiedlichen Kriterien. Sie kann über die Ausdehnung, das Alter und das Geschlecht, nach dem Schweregrad oder der Histologie erfolgen. Klinisch bewährt hat sich die Einteilung in eine primäre und eine sekundäre Osteoporose, da sich hieraus direkte therapeutische Konsequenzen ableiten lassen. Unter der primären Osteoporose versteht man die idiopathische, postmenopausale und altersassoziierte Osteoporose. Sie stellt die weitaus häufigste Erscheinungsform der Osteoporose dar und hält einen Anteil von rund 95\% aller Osteoporoseformen. Sekundäre Osteoporosen sind die Folge anderer Erkrankungen des Stoffwechsels oder des Skelettsystems oder aber die Folge langjähriger Einnahme bestimmter Medikamente. Typische
Beispiele hierfür sind die Inaktivitätsosteoporose, die Osteoporose bei Hyperparathyreoidismus oder die kortisoninduzierte Osteoporose. Allen Formen gemeinsam sind die messbare Reduktion der Knochendichte sowie die damit einhergehende hohe Frakturanfälligkeit.

Jede zweite Frau und jeder fünfte Mann erleidet im Laufe des Lebens eine osteoporosebedingte Fraktur [9]. Dabei ist bei Frauen die Wahrscheinlichkeit an den Folgen dieser Fraktur zu versterben höher, als an den Folgen von Brust- und Ovarialkarzinom zusammengenommen [8]. In der Bundesrepublik wird derzeit mit rund 6 Millionen Osteoporosepatienten gerechnet.

Die Betreuung dieser Patienten verursacht allein in Deutschland geschätzte Kosten von rund 10 Milliarden Euro jährlich, mit steigender Tendenz [7].

In den USA werden ungefähr 50 Millionen Dollar täglich für die Versorgung osteoporoseassoziierter Frakturen ausgegeben. Die Osteoporose gehört damit zu den 10 wichtigsten und teuersten Volkskrankheiten.

In den meisten Fällen wird eine bestehende Osteoporose erst durch das Auftreten von Frakturen klinisch manifest. Da Patienten mit Frakturen in Deutschland in einer unfallchirurgischen oder orthopädischen Klinik behandelt werden, kommt dem Unfallchirurgen und Orthopäden eine Schlüsselrolle in der Diagnostik und Therapieeinleitung dieser Erkrankung zu [2]. In einer aktuellen Untersuchung aus der eigenen Klinik konnte gezeigt werden, dass die Inzidenz reduzierter Knochendichte (Osteoporose und Osteopenie) bei Patienten über 50 Jahren und extravertebralen Frakturen rund 96\% beträgt. Eine manifeste Osteoporose wiesen $72 \%$ auf [15]. Da Personen höheren Alters inzwischen nahezu die Hälfte aller stationär behandelten Patienten mit Extremitätenfrakturen darstellen, beträgt 
der Anteil der osteoporoseassoziierten Frakturen in der Unfallchirurgie mittlerweile zwischen 40 und $45 \%$.

Das Ziel einer leitliniengerechten Diagnostik und Therapie der Osteoporose ist, Folgefrakturen zu verhindern, die Lebensqualität der betroffenen Patienten zu verbessern und die Kosten für die sozialen Sicherungssysteme zu senken.

\section{Hauptteil}

Diagnostik

Osteoporose an sich tut zunächst nicht weh. Erst wenn Frakturen auftreten wird sie symptomatisch.

Die diagnostischen Maßnahmen bei der Osteoporose dienen somit vor allem der Einschätzung des Frakturrisikos sowie dem Ausschluss anderer Skeletterkrankungen.

\section{Anamnese}

Ein Großteil der Patienten sucht den Arzt aufgrund akuter oder chronischer Rückenschmerzen auf. Die Ursachen hierfür reichen von Muskelverspannungen über Fibromyalgie und Bandscheibenvorfall bis hin zu Knochenmetastasen und einem akuten Myokardinfarkt. Sie bedürfen einer sorgfältigen Abklärung durch den behandelnden Arzt.

Im Bezug auf die Osteoporose ist das Auftreten von Rückenschmerzen bereits ein Anzeichen für stattgefundene Wirbelkörperfrakturen. Eine gewissenhafte Anamnese unter Einbeziehung von Risikofaktoren, Lebensstil, Ernährung und Medikamenteneinnahme lenkt in den meisten Fällen den Verdacht in die richtige Richtung.

\section{Körperliche Untersuchung}

Bei der körperlichen Untersuchung wird besonderer Wert auf die Detektion indirekter Hinweise für bisher nicht diagnostizierte Wirbelkörperfrakturen gelegt. Hierbei spielen die Körpergröße und deren Abnahme, die Statik und Körperhaltung, Muskelverspannungen, Klopfschmerzhaftigkeit und Beweglichkeit der Wirbelsäule sowie der Nachweis einer Kyphose oder Skoliose eine wichtige Rolle. Die klassischen und auf den ersten Blick erkennbaren Zeichen einer Osteoporose lassen sich auf die zum Teil erhebliche Größenabnahme $(>4 \mathrm{~cm})$ des Achsenskeletts zurückführen. Durch die Rumpfverkürzung kann der untere

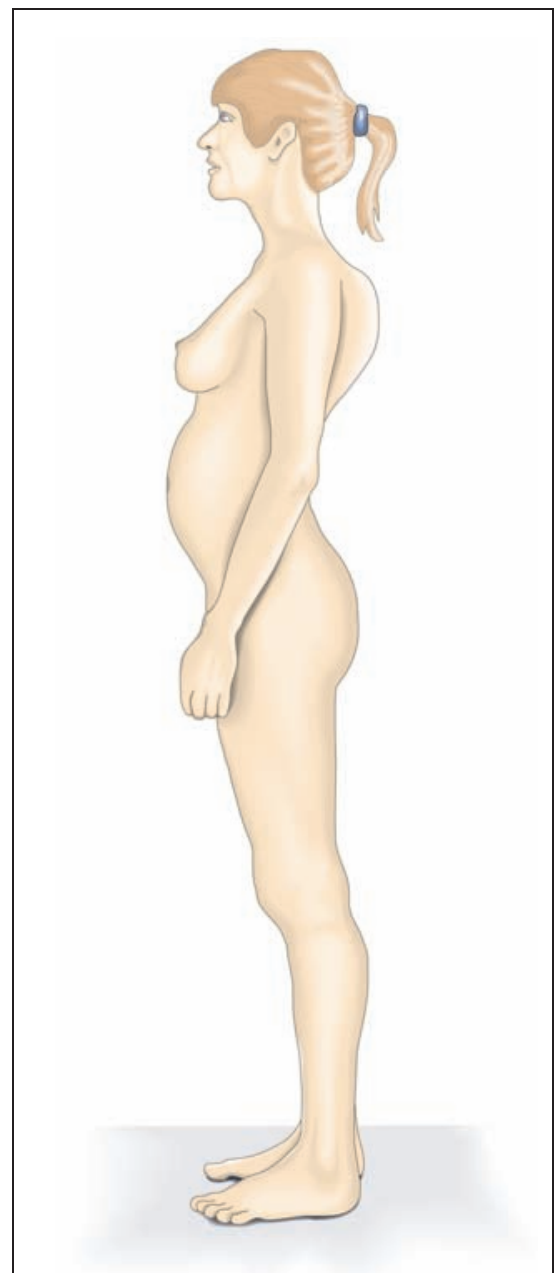

Abb.1 Körperdeformitäten bei Osteoporose (Witwenbuckel, Osteoporose-Bäuchlein), hervorgerufen durch Höhenminderung der Wirbelkörper.

Rippenbogen sogar den Beckenkamm schmerzhaft berühren. Es kommt dabei zu charakteristischen Hautfalten an Rücken und Flanken, dem sog. „Tannenbaumphänomen“ sowie zur Vorwölbung des Bauches, dem sog. „OsteoporoseBäuchlein“. Eine weitere Folge der Höhenminderung der Wirbelkörper ist das „Baastrup-Zeichen“ („kissing-spine“), ausgelöst durch die schmerzhafte Berührung benachbarter Dornfortsätze. Durch die Entwicklung einer ausgeprägten Kyphose verlagert sich der Körperschwerpunkt weit nach vorne. Weiteres typisches Zeichen ist der sog. „Witwenbuckel“, der durch Keilwirbelbildung vor allem an der Brustwirbelsäule hervorgerufen wird (Abb.1).

Die Osteoporose verändert so die gesamte Körperstatik, verstärkt die Gangunsicherheit und erhöht die Sturzneigung der betroffenen Patienten.
Laboruntersuchung

Die primäre Osteoporose führt nicht $\mathrm{zu}$ Veränderungen der üblichen Laborparameter in Blut oder Urin. Zur Abklärung sekundärer Osteoporoseformen $(<5 \%)$ sind wenige Routineuntersuchungen von Blut, Serum und Urin notwendig.

Basislabor:

- Blutkörperchensenkung oder CRP

- Kleines Blutbild

- Kalzium und Phosphat (Serum)

- Alkalische Phosphatase (Serum)

- Glukose(Serum/Urin)

- Transaminasen und Gamma-GT (Serum)

- Kreatinin (Serum)

\section{Knochenmarker}

Die Parameter des Knochenumbaus eignen sich zwar nicht zur Diagnostik der Osteoporose, jedoch können sie einige klinische Fragestellungen beantworten. Dazu gehören die Rate des Knochenumbaus, die Frage nach dem Risiko für eine osteoporotische Fraktur sowie die Effektivitätsbeurteilung einer laufenden Osteoporosetherapie.

Im Rahmen der Knochenneubildung oder Knochenresorption werden von Osteoblasten und Osteoklasten Enzyme produziert, deren Aktivität laborchemisch ermittelt werden kann (z.B. tartratresistente saure Phosphatase, Osteokalzin, knochenspezifische alkalische Phosphatase).

Außerdem lassen sich Metaboliten der Knochenmatrix, die während des Abbaus oder der Neubildung anfallen (z. B. Crosslinks, Prokollagen-I, N-und C-Telopeptide des Typ-I-Kollagens), messen. Diese beiden Substanzgruppen können eine radiologische Knochendichtemessung zwar nicht ersetzen, geben aber unter anderem Hinweise auf das zukünftige Ausmaß des Knochenschwundes und das drohende Frakturrisiko. Die Klärung dieser speziellen klinischen Fragen ist jedoch dem Osteologen vorbehalten.

\section{Knochenbiopsie}

Eine Knochenbiopsie ist nach heutigen Gesichtspunkten nur noch zum Ausschluss anderer Erkrankungen des Knochen oder des Knochenmarkes indiziert.

Hierzu gehören sekundäre Formen der Osteoporose und maligne metastasierende Prozesse. Der Verdacht einer Minerali- 


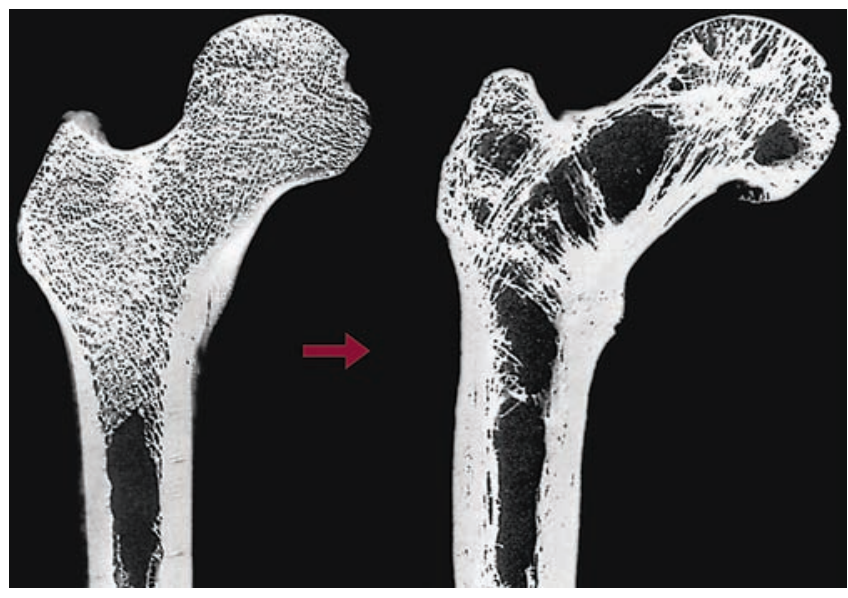

Abb. 2 Rarefizierung der Spongiosa am Beispiel des proximalen Femurs (anatomisches Präparat). Links gesunder Femur, rechts Femur mit Osteoporose (zur Verfügung gestellt von R. Bartl).

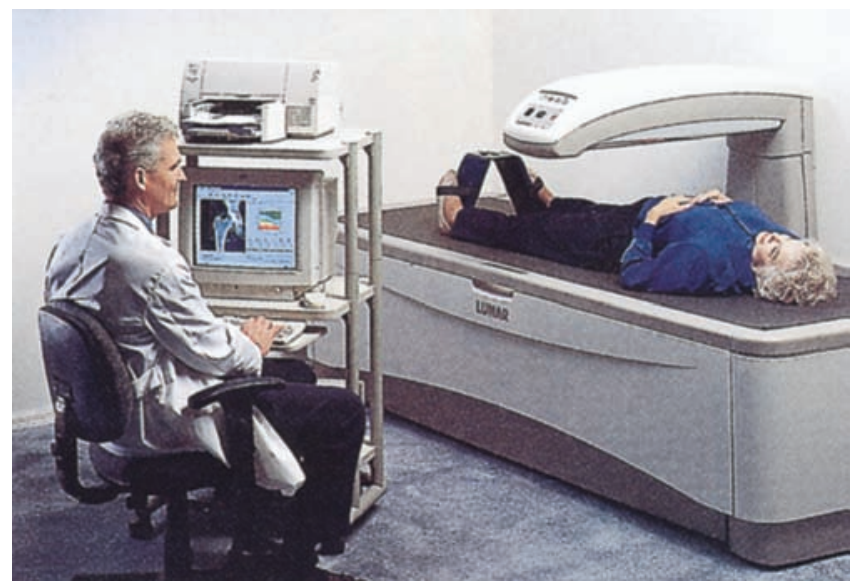

Abb.3 Moderne Durchführung der Knochendichtemessung mittels DXA an Lendenwirbelsäule und Hüfte. Vorteile: schnell, kostengünstig (30-50€), geringe Strahlenbelastung (1-3 mRem). sationsstörung kann mittels Knochenbiopsie eindeutig abgeklärt werden.

\section{Bildgebung}

Den größten Stellenwert in der apparativen Osteoporosediagnostik haben zweifelsohne die bildgebenden Verfahren. An erster Stelle und von der WHO empfohlen steht hierbei die Kombination aus konventioneller Radiographie und Knochendichtemessung mittels Dual-Xray-Absorptiometrie (DXA) [16].

Obwohl für eine Frühdiagnose der Osteoporose nicht geeignet, lassen sich mit Röntgenaufnahmen des Skeletts abgelaufene Frakturen oder Einbrüche der Wirbelkörper nach Art und Lage entdecken und quantifizieren. Die Osteoporose führt zunächst zu einer Rarefizierung der Spongiosa und erst später zu einer Verschmälerung der Kortikalis (Abb.2). Ein gesunder Wirbelkörper weist im konventionellen Röntgenbild neben seiner Rahmenstruktur horizontale und vertikale Akzentuierungen als Ausdruck seiner enthaltenen Spongiosa auf. Mit zunehmender Knochendichteminderung verblassen zuerst die horizontalen und erst später die vertikalen Zeichnungen. Auf der Höhe der Erkrankung präsentiert sich der betroffene Wirbelkörper radiologisch nur noch als scharf umrandetes Rechteck („empty-box“). Neben diesen radiologischen Zeichen der Osteoporose dient die konventionelle Röntgenaufnahme der Wirbelsäule vor allem der Verifizierung der DXA-Messung. Degenerative Veränderungen wie osteophytäre Anbauten oder Aortenkalk können in der DXA zu falsch hohen Werten führen und werden durch das konventionelle Röntgenbild objektiviert.
Die DXA-Messung ist heute die ausgereifteste und populärste Messmethode.

Sie wird von der WHO favorisiert und stellt die Grundvoraussetzung einer standardisierten Osteoporosediagnostik dar. Neben den Vorteilen einer sehr geringen Strahlenbelastung (1-3 mREM) und einer unkomplizierten, schnellen und preiswerten Durchführbarkeit stellt sie keine Belastung für den Patienten dar (Abb.3). Sie misst sehr genau (Richtigkeit 2-6\%, Präzision 1-3\%) und ist dadurch ideal für Kontrollmessungen geeignet [1]. Die Messung erfolgt definitionsgemäß an Hüfte und LWS. Ermittelt wird der sog. „T-Score“. Es handelt sich dabei um den Vergleich der Dichtewerte der gemessenen Person mit denen eines gesunden jungen Erwachsenen (Vergleich mit der maximalen Knochendichte), angegeben als Standardabweichung (Abb.4).

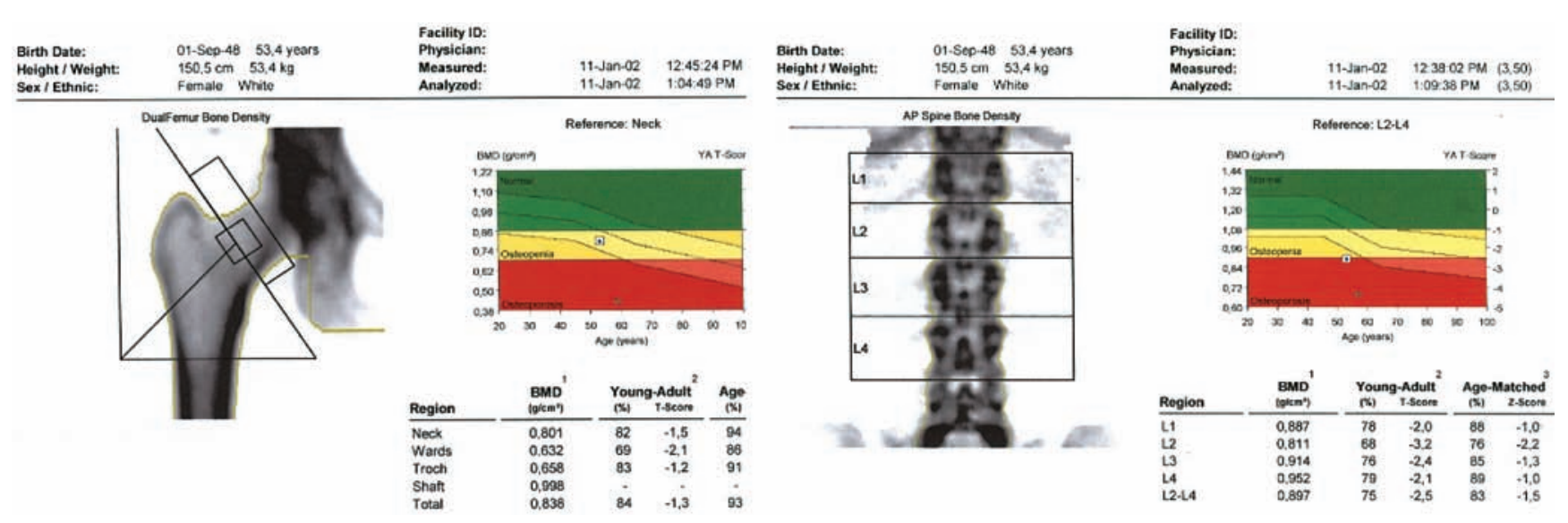

Abb. 4 DXA Messung an Lendenwirbelsäule und Schenkelhals und Ausdruck der errechneten Daten eines Patienten mit Osteoporose (Wirbelsäule). $\mathrm{BMD}=$ Knochendichte, $\mathrm{T}$-Score = Vergleich der Knochendichte mit jungen gesunden Erwachsenen, angegeben als Standardabweichung (SD=) . 
Tab. 1 WHO-Definition der Osteoporose anhand der Ergebnisse der Knochendichtemessung (DXA). SD = Standardabweichung

\begin{tabular}{ll} 
Bewertung & T-Score \\
\hline Normalbefund & $\geq-1 \mathrm{SD}$ \\
Osteopenie & -1 bis $-2,5 \mathrm{SD}$ \\
Osteoporose (messtechnisch) & $\leq-2,5 \mathrm{SD}$ \\
Osteoporose (manifest) & $\leq-2,5 \mathrm{SD}$ plus Fraktur
\end{tabular}

\begin{tabular}{ll}
\hline Tab. 2 & WHO-Stufenschema zur medikamentösen Analgesie \\
\hline Stufe & Stoffklasse \\
\hline I & $\begin{array}{l}\text { Nichtopioidanalgetika (z. B. Ibuprofen, Naproxen, Diclofenac, Celecoxib, Rofecoxib, } \\
\text { Metamizol). Bei Insuffizienz der Stufe I wird ein schwaches Opioid ergänzt }\end{array}$ \\
II & $\begin{array}{l}\text { Schwach wirkende Opioide (z. B. Codein, Dihydrocodein, Tramadol, Tilidin/Nal- } \\
\text { oxon). Bei Insuffizienz der Stufe II wird das schwache durch ein starkes Opioid } \\
\text { ersetzt }\end{array}$ \\
III $\quad \begin{array}{l}\text { Stark wirkende Opioide (z. B. Hydromorphon, Morphin, Fentanyl, L-Methadon, } \\
\text { Oxycodon, Buprenorphin) }\end{array}$
\end{tabular}

Anhand der ermittelten T-Werte erfolgt definitionsgemäß die Diagnosestellung der Osteoporose (Tab.1). Der T-Wert wurde deshalb gewählt, da sich zeigte, dass schon die Verminderung der Knochendichte um 10\% mit einer Verdopplung des Frakturrisikos im Bereich der Wirbelsäule und einer Verdreifachung im Bereich des Oberschenkelhalses einhergeht.

Weitere Diagnosewerkzeuge können die quantitative Computertomographie (QCT), die periphere quantitative Computertomographie (pQCT) und die quantitative Ultraschallmessung (QUS) an Ferse und Fingern darstellen. Die QCT erlaubt eine getrennte Messung von Spongiosa und Kortikalis und eignet sich am besten um einen frühen Verlust an trabekulärem Knochen der Wirbelsäule zu entdecken. Jedoch geht sie mit einer weit höheren Strahlenbelastung (100-300 mRem) einher, dauert ca. 20 Minuten, ist kostenintensiver und für Verlaufskontrollen nur eingeschränkt geeignet. Die pQCT hat klinisch keine große Bedeutung. Ihre Werte lassen sich nicht unkritisch auf das Gesamtskelett übertragen. Jedoch kann sie bei speziellen Fragestellungen zum Einsatz kommen und erlaubt eine dreidimensionale Darstellung der Knochenstruktur z.B. von Tibia und Radius.

Die quantitative Ultraschallmessung stellt zum heutigen Stand der Technik noch keine sinnvolle Alternative zu den oben genannten Messverfahren dar. Sie ist aber als Screeningmethode zur Beur- teilung des Frakturrisikos von Wert, da sie sehr einfach und ohne Strahlenbelastung durchzuführen ist.

Die Magnetresonanztomographie (MRT) hat in der Osteoporosediagnostik keinen Stellenwert, sie eignet sich aber zur Darstellung entzündlicher oder tumoröser Knocheninfiltrate und dient der Differenzialdiagnostik.

\section{Therapie}

Die zeitgemäße Therapie der Osteoporose ruht auf mehreren Säulen. Neben einer Reduktion der Risikofaktoren und der physikalischen Therapie steht dabei am Anfang eine suffiziente Schmerztherapie, gefolgt von der sog. Basistherapie. Weiterhin steht uns heute eine Reihe von Medikamenten zur Verfügung, die im Wesentlichen in zwei Gruppen aufgeteilt werden können:

Antiresorptive Substanzen: Raloxifen, Bisphosphonate, Kalzitonine, Kalzium, Vitamin D, Vitamin-D-Metaboliten, Statine, Östrogene, Östrogen/Gestagen und Tibolon.

Osteoanabole Substanzen: Parathormon, Fluoride, Strontium, Anabolika und Testosteron.

Die Effektivität der genannten Substanzen wird heute nicht mehr über die messtechnische Zunahme der Knochendichte, sondern allein über die Reduktion des Frakturrisikos bestimmt. Die Entschei- dung für ein Medikament wird aber nach wie vor von verschiedensten Motiven beeinflusst. Diese reichen von subjektiven Abwägungen des behandelnden Arztes bis hin zu Budgetproblemen.

Die moderne Therapie der Osteoporose sollte sich jedoch nach dem Evidenzgrad der nachgewiesenen Wirksamkeit des jeweiligen Präparates richten.

\section{Schmerztherapie}

Der Schmerz einer Brustwirbelfraktur kann durchaus die Schmerzintensität des Vernichtungsschmerzes bei einem Myokardinfarkt erreichen.

Eine adäquate und konsequente Analgesie nach den Grundprinzipien der medikamentösen Therapie und dem Stufenschema der WHO ist zur Lebensqualitätverbesserung obligat (Tab.2).

\section{Basistherapie}

Auch wenn die Basistherapie mit Kalzium und Vitamin $\mathrm{D}_{3}$ in der aktuellen Literatur kontrovers diskutiert wird, existieren mehrere Studien, die eine signifikante Senkung des Frakturrisikos unter der oralen Substitution mit 800 - 1200 mg/d Kalzium und $700-1000 \mathrm{IE} / \mathrm{d}$ Vitamin $\mathrm{D}_{3}$ belegen [5]. Entscheidende Argumente für eine solche Basistherapie sind der nachgewiesene Mangel dieser Substanzen in der alten Bevölkerung [10], sowie eine unter Vitamin-D3-Medikation nachweisbare deutliche Senkung der Sturzhäufigkeit. Postuliert wird hierbei eine Stärkung des muskulären Halteapparates durch eine vermehrte Aufnahme von Kalzium in das sarkoplasmatische Retikulum.

\section{Hormontherapie}

In einer großen Zahl von Studien konnte der positive Einfluss einer Östrogen-, Östrogen-/Gestagenkombination auf die Zunahme der Knochendichte belegt werden. In einer umfangreichen amerikanischen Studie konnte im Jahr 2002 an 16600 postmenopausalen Frauen unter Hormonersatztherapie (HRT) eine signifikante Reduktion vertebraler und extravertebraler Frakturen gezeigt werden. Jedoch führte die HRT im Vergleich zur Kontrollgruppe zu einer Steigerung des Herzinfarkt- und Schlaganfallrisikos sowie zu einer deutlichen Zunahme der Brustkrebsinzidenz. Die Risiko-NutzenAbwägung führte sogar zu einem vorzeitigen Abbruch dieses Studienarms [13]. 
Unter Berücksichtigung anderer effektiver Medikamente ist die Verwendung der HRT zur Prävention und Therapie der Osteoporose heute nicht mehr nötig.

Sie ist derzeit nur noch zur Behandlung klimakterischer Beschwerden indiziert und sollte auf wenige Jahre beschränkt werden.

\section{Bisphosphonate}

Die Einführung der Bisphosphonate vor rund 30 Jahren ermöglichte neue Ansätze in der Osteoporosetherapie. Ihre Wirksamkeit beruht auf einer Anreicherung ausschließlich im Bereich der Resorptionslakunen unterhalb der Osteoklasten und einer damit verbundenen direkten enzymatischen Hemmung der Osteoklastenaktivität über eine Steigerung der Zellapoptose. In tierexperimentellen Untersuchungen konnte dabei eine Verzögerung der normalen Knochenbruchheilung nicht beobachtet werden.

Die antiresorptive Potenz der Bisphosphonate hemmt damit den Knochenabbau und führt zu einer positiven Knochenbilanz. In den aktuellen Leitlinien des Dachverbandes Osteologie (DVO) spielen zwei Vertreter dieser Stoffgruppe die größte Rolle: Alendronat (Fosamax ${ }^{\circledR}$ ) und Risedronat $\left(\right.$ Actonel $^{\circledR}$ ). Beide Substanzen haben ihre Wirksamkeit bezüglich einer Senkung der Frakturhäufigkeit in großen randomisierten Studien bewiesen. Dabei erreichte Alendronat $70 \mathrm{mg}$ wöchentlich über 12 Monate signifikant höhere Knochendichtewerte an Wirbelsäule und Oberschenkel als Risedronat $5 \mathrm{mg}$ täglich [12]. Die Therapie mit $10 \mathrm{mg}$ Alendronat pro Tag (70 mg/Woche) führte nach einjähriger Anwendung zu einer Reduktion klinischer Wirbelkörperfrakturen von 59\% und nach 18 Monaten zu einer Reduktion von Oberschenkelhalsfrakturen von 63\% gegenüber der Plazebo-Gruppe. Auch Schmerzreduktion und Zunahme der Mobilität waren unter Alendronat nachweisbar [3]. Weitere Vertreter der Bisphosphonate wie Ibandronat $\left(\right.$ Bandronat $\left.^{\circledR}\right)$, Clodronat (Clodron Hexal $^{\circledR}$ ), Pamidronat (Ebedronat ${ }^{\circledR}$ ) und Zoledronat (Zometa ${ }^{\circledR}$ ) wurden bezüglich ihrer Wirksamkeit bereits Erfolg versprechend getestet, sind aber für die Therapie der Osteoporose noch nicht zugelassen und kommen derzeit nur in Osteoporosezentren zum Einsatz. Auch die intravenöse Applikation von Pamidronat, Ibandronat und Zoledronat besitzt noch keine offizielle Zulassung für die Behandlung der Osteoporose.

\section{Raloxifen (Evista ${ }^{\circledR}$ )}

Dieser „Selective Estrogen Receptor Modulator" (SERM) gehört zu den ersten Vertretern dieser Stoffgruppe, besitzt die Zulassung zur Prävention und Therapie der Osteoporose und ist ebenfalls, wie die Bisphosphonate Alendronat und Risedronat, Bestandteil der aktuellen DVOLeitlinien. Er interagiert direkt mit dem Östrogenrezeptor und führt durch seine östrogenagonistische Wirkung auf Knochen- und Lipidstoffwechsel zu einem antiresorptiven Effekt. In der größten internationalen klinischen Studie, der MORE-Studie aus dem Jahr 1999, konnte eine Senkung des Risikos für klinische Wirbelkörperfrakturen um 68\% gegenüber Plazebo gezeigt werden [14]. Somit stellt Raloxifen eine wichtige Bereicherung für die Behandlung der Osteoporose dar.

\section{Parathormon}

Parathormon (PTH) ist ein Polypeptid aus 84 Aminosäuren, es mobilisiert Kalzium und Phosphat aus dem Knochen und regt die Synthese von aktivem Vitamin $\mathrm{D}$ in der Niere an. Der kleine Bruder Teriparatid (rhPTH 1-34) (Forsteo $^{\circledR}$ ) besteht nur aus den ersten 34 Aminosäuren des Parathormons. Er aktiviert Osteoblasten auf allen Knochenoberflächen und führt zu einer Erhöhung von Knochendichte, Knochenbelastbarkeit und Verknüpfung der Knochenbälkchen. In einer 2-Jahresstudie bei postmenopausalen Frauen mit Osteoporose senkte subkutan verabreichtes Teriparatid die Rate neuer Frakturen um 65-70\% [11]. Obwohl Teriparatid derzeit noch nicht in den Leitlinien der DVO enthalten ist, wird es mit hoher Wahrscheinlichkeit bei der Neufassung Berücksichtigung finden.

\section{Strontium (Protelos ${ }^{\circledR}$ )}

Ebenso wie für Teriparatid enthalten die aktuellen DVO-Leitlinien keine Empfehlung für Strontium. Strontium wirkt gleichzeitig osteoanabol und antiresorptiv und hat seine Wirksamkeit in mehreren Studien bewiesen. Ob es aber zukünftig in der Osteoporosetherapie eingesetzt werden kann, müssen kommende Studien im Hinblick auf die Senkung des Frakturrisikos klären.

\section{Weitere Therapie}

Kalzitonin und Fluoride werden den heutigen Ansprüchen an moderne Osteoporosetherapie-Studien nicht gerecht. Die groß angelegte PROOF-Studie („Prevent Recurrence of Osteoporotic Fractures“) mit Kalzitonin führte aufgrund erheblicher methodischer Mängel zu einer $\mathrm{Ab}$ bruchsrate von 59\% innerhalb von 5 Jahren. Ebenso wenig konnte die Wirkung der Fluoride in Studien ausreichend belegt werden.

Weitere Substanzen wie Leptin, Osteoprotegerin, Wachstumsfaktoren, Statine, Anabolika, Tetrazykline (CMTs), Vitamin $\mathrm{K}$, Tibolon und neue SERMs werden getestet, haben aber noch keinen Eingang in die Therapie der Osteoporose gefunden.

\section{Schlussfolgerung (Fazit für die Praxis)}

Die Osteoporose und die damit vergesellschafteten Frakturen haben großen Einfluss auf die Lebensqualität des Einzelnen und die Kostenentwicklung im Gesundheitswesen. Dies zu akzeptieren und in laufende Therapieentscheidungen mit einzubeziehen stellt den größten Meilenstein auf dem Weg zu einer zeitgemäßen und effektiven Therapie dieser Erkrankung dar. Ist diese Hürde genommen, kann eine sinnvolle Diagnostik und Therapie anhand der geltenden Leitlinien erfolgen. Dem Unfallchirurgen kommt hierbei eine wichtige Schlüsselfunktion $\mathrm{zu}$. Eine konsequente Therapie wird durch die Vermeidung von Erst- und die erfolgreiche Verhinderung von Folgefrakturen belohnt.

\section{Literatur}

${ }^{1}$ Bartl R. Osteoporose - Prävention, Diagnostik, Therapie. Thieme, Stuttgart New York 2001

2 Bartl R, Bartl C, Mutschler W. Diagnostik und Therapie der Osteoporose. Unfallchirurg 2003; 106: 526 - 541

3 Black DM, Thompson DE, Bauer D et al. Fracture Risk Reduction with Alendronate in Women with Osteoporosis: The Fracture Intervention Trial. Osteoporos Int 2000; 85: $4118-4124$

${ }^{4}$ www.boneandjointdecade.org/

${ }^{5}$ Dawson-Huges B, Harris SS, Krall EA et al. Effect of Calcium and Vitamin D Supplementation on Bone Density in Men and Women 65 Years of Age or Older. N Eng J Med 1997; 337: $670-676$

${ }^{6}$ Report of a WHO Scientific Group, Prevention and Management of Osteoporosis, WHO Technical Report Series (2003) No 921

Götte S, Dittmar K. Epidemiologie und Kosten der Osteoporose. Orthopäde 2001; 30: 402- 404

${ }^{8}$ Hanley DA, Josse RG. Prevention and management of osteoporosis: consensus statements from the Scientific Advisory Board of the Osteoporosis Society of Canada. CMAJ 1996; 155: 921 -923 
${ }^{9}$ Jones G, Nguyen T, Sambrook PN et al. Symptomatic fracture incidence in elderly men and women: The Dubbo Osteoporosis Epidemiology Study (DOES). Osteoporos Int 1994; 4: 277-282

${ }^{10}$ Lips P. Vitamin D deficiency and secondary hyperparathyreoidism in the elderly: consequences for bone loss and fractures and therapeutic implications. Endocrine Reviews 2001; 22: 477-501

${ }^{11}$ Neer R, Arnaud C, Zanchetta J et al. Effect of parathyroid hormone $(1-34)$ on fractures and bone mineral density in postmenopausal women with osteoporosis. N Engl J Med 2001; 344: 1434-1441

${ }^{12}$ Reginster JY, Minne HW, Sorensen OH et al. Randomized Trial of the Effect of Risedronate on Vertebral Fractures in Women with Established Postmenopausal Osteoporosis. Osteoporos Int. 2000; 11: 83-91
${ }^{13}$ Rossouw JE, Anderson GL, Prentice RL et al. Risks and benefits of estrogen plus progestin in healthy postmenopausal women (Women's Health Initiative Group). JAMA 2002; 288: $321-333$

${ }^{14}$ Siris E, Adachi JD, Lu Y et al. Effects of raloxifene on fracture severity in postmenopausal women with osteoporosis: results from the MORE study. Multiple Outcomes of Raloxifene Evaluation. Osteoporos Int. 2002; 13: 907-13

${ }^{15}$ Vogel T, Dobler T, Bitterling H et al. Osteoporose in der Unfallchirurgie: Prävalenz und Management. Unfallchirurg 2005; 108: 356-364

${ }^{16}$ World Health Organization (1994). Assessment of fracture risk and its application to screening for postmenopausal osteoporosis. Technical Report Series. WHO, Genf
Tobias Vogel

Assistenzarzt

Leiter der Arbeitsgruppe Klinisches Osteoporose-Management

Philip Kampmann

Assistenzarzt

Prof. Dr. med. Wolf Mutschler

Direktor

Chirurgische Klinik und Poliklinik der Universität München-Innenstadt

Nussbaumstraße 20

D-80336 München

Prof. Dr. med. Klaus Jürgen Pfeifer

Geschäftsführender Oberarzt

Institut für Klinische Radiologie der Universität München-Innenstadt

Nussbaumstraße 20

D-80336 München

Prof. Dr. med. Rainer Bartl

Leiter des Bayerischen Osteoporosezentrums

Medizinische Klinik III Klinikum der Universität München-Großhadern Marchioninistraße 15

D-81377 München 